Journal of Mathematics and Statistics 8 (1): 129-135, 2012

ISSN 1549-3644

(C) 2012 Science Publications

\title{
Numerical Solution for 2D European Option Pricing Using Quarter-Sweep Modified Gauss-Seidel Method
}

\author{
${ }^{1}$ Koh Wei Sin, ${ }^{1}$ Jumat Sulaiman and ${ }^{2}$ Rasid Mail \\ ${ }^{1}$ Mathematics with Economics Programme, School of Science and Technology, \\ ${ }^{2}$ Accountancy Programme, School of Business and Economics, \\ University Malaysia Sabah, 88400 Kota Kinabalu, Sabah, Malaysia
}

\begin{abstract}
Problem statement: This study presents the numerical solution of two-dimensional European option pricing problem based on Quarter-Sweep Modified Gauss-Seidel (QSMGS) iterative method. In fact, the pricing of European option with two-underlying assets can be governed by twodimensional Black-Scholes Partial Differential Equation (PDE). Approach: The PDE needs to be discretized by using full-, half- and quarter-sweep second-order Crank-Nicolson schemes to generate a system of linear equations. Then, the Modified Gauss-Seidel, a preconditioned iterative method is applied to solve the generated linear system. Results: In order to examine the effectiveness of QSMGS method, several numerical experiments of Full-Sweep Gauss-Seidel (FSGS), Half-Sweep Gauss-Seidel (HSGS) and Quarter-Sweep Gauss-Seidel (QSGS) methods are also included for comparison purpose. Thus, the numerical experiments show that the QSMGS iterative method is the fastest in computing as well as having the least number of iterations. In the error analysis, QSMGS method shows good and consistent results. Conclusion: Finally, it can be concluded that QSMGS method is superior in increasing the convergence rate.
\end{abstract}

Key words: Modified Gauss-Seidel, quarter-sweep iteration, two-dimensional Black-Scholes PDE, Crank-Nicolson scheme

\section{INTRODUCTION}

Option is a financial derivative which gives the holder the right to trade the underlying asset by a certain date for a certain price. In trading the option, the right to buy is known as call option while the vice versa is put option. Black and Scholes (1973) and Merton (1973) derived the Black-Scholes Partial Differential Equation (PDE) for option pricing which earned them the 1997 Nobel Prize in Economics. In this study, we focus on a two-dimensional Black-Scholes PDE as follows Eq. 1 (Stulz, 1982; Jeong et al., 2009):

$$
\begin{aligned}
\frac{\partial \mathrm{v}}{\partial \mathrm{t}}= & -\frac{1}{2} \sigma_{1}^{2} \mathrm{~s}_{1}^{2} \frac{\partial^{2} \mathrm{v}}{\partial \mathrm{s}_{1}^{2}}-\frac{1}{2} \sigma_{2}^{2} \mathrm{~s}_{2}^{2} \frac{\partial^{2} \mathrm{v}}{\partial \mathrm{s}_{2}^{2}} \\
& -\rho \sigma_{1} \sigma_{2} \mathrm{~s}_{1} \mathrm{~s}_{2} \frac{\partial^{2} \mathrm{v}}{\partial \mathrm{s}_{1} \partial \mathrm{s}_{2}}-\mathrm{rs}_{1} \frac{\partial \mathrm{v}}{\partial \mathrm{s}_{1}}-\mathrm{rs}_{2} \frac{\partial \mathrm{v}}{\partial \mathrm{s}_{2}}-\mathrm{rv}
\end{aligned}
$$

Where:

$\mathrm{v}=$ Value of the option

$\mathrm{t}=$ Time $\mathrm{s}_{1}=$ First asset's prices

$\mathrm{s}_{2}=$ Second asset's price

$\sigma_{1}=$ Volatility of the first asset's price

$\sigma_{2}=$ Volatility of the second asset's price

$\rho=$ Correlation between the two assets' price

$\mathrm{r}=$ Risk free interest rate

A European call option on the maximum of two underlying assets is evaluated by solving the twodimensional Black-Scholes PDE. The final time condition for this problem is Eq. 2 (Stulz, 1982; Haug, 2007):

$$
\mathrm{v}\left(\mathrm{s}_{1}, \mathrm{~s}_{2}, \mathrm{~T}\right)=\max \left(\max \left(\mathrm{s}_{1}, \mathrm{~s}_{2}\right)-\mathrm{K}, 0\right)
$$

Where:

$\mathrm{K}=$ Exercise price

$\mathrm{T}=$ Maturity time

Besides that, the boundary conditions are Eq. 3-6:

$\mathrm{v}\left(\mathrm{s}_{1}, 0, \mathrm{t}\right)=\mathrm{s}_{1} \mathrm{~N}\left(\mathrm{~d}_{1}\right)-\mathrm{Ke}^{-\mathrm{rt}} \mathrm{N}\left(\mathrm{d}_{2}\right)$

Corresponding Author: Koh Wei Sin, Mathematics with Economics Programme, School of Science and Technology, University Malaysia Sabah, 88400 Kota Kinabalu, Sabah, Malaysia 


$$
\begin{aligned}
& \mathrm{v}\left(0, \mathrm{~s}_{2}, \mathrm{t}\right)=\mathrm{s}_{2} \mathrm{~N}\left(\mathrm{~d}_{1}\right)-\mathrm{Ke}^{-\mathrm{rt}} \mathrm{N}\left(\mathrm{d}_{2}\right) \\
& \mathrm{v}\left(\mathrm{S}_{1}, \mathrm{~S}_{2}, \mathrm{t}\right)=\mathrm{S}_{1}-\mathrm{Ke}^{-\mathrm{rt}} \\
& \mathrm{v}\left(\mathrm{s}_{1}, \mathrm{~S}_{2}, \mathrm{t}\right)=\mathrm{S}_{2}-\mathrm{Ke}^{-\mathrm{rt}}
\end{aligned}
$$

Where:

$$
\begin{aligned}
& \mathrm{d}_{1}=\frac{\ln \left(\frac{\mathrm{s}}{\mathrm{K}}\right)+\left(\mathrm{r}+\frac{\sigma^{2}}{2}\right)(\mathrm{T}-\mathrm{t})}{\sigma \sqrt{\mathrm{T}-\mathrm{t}}} \\
& \mathrm{d}_{2}=\frac{\ln \left(\frac{\mathrm{s}}{\mathrm{K}}\right)+\left(\mathrm{r}-\frac{\sigma^{2}}{2}\right)(\mathrm{T}-\mathrm{t})}{\sigma \sqrt{\mathrm{T}-\mathrm{t}}}=\mathrm{d}_{1}-\sigma \sqrt{\mathrm{T}-\mathrm{t}}
\end{aligned}
$$

Where:

$\mathrm{N}$ = Cumulative normal distribution

$\mathrm{S}_{1}=$ Maximum of $\mathrm{s}_{1}$

$\mathrm{S}_{2}=$ Maximum of $\mathrm{s}_{2}$

Actually, the boundary conditions when one of the asset prices is zero as shown in 3,4 are obtained by using Black-Scholes formula (Black and Scholes, 1973).

The main objective of this study is to introduce Quarter-Sweep Modified Gauss-Seidel (QSMGS) method in solving the two-dimensional Black-Scholes PDE in European option pricing. Previously, QSMGS method had been used to solve the one-dimensional Black-Scholes PDE in European (Koh and Sulaiman, 2009) and American option pricing (Koh et al., 2010a). The results obtained have pointed out that QSMGS iterative method has a better convergence rate. For this manner, it is more motivating to study QSMGS in solving two-dimensional problem. Nevertheless, to discretize the PDE in Eq. 1, we will apply the quartersweep Crank-Nicolson approach. The Crank-Nicolson discretization scheme has second order accuracy and stability plus it is very famous for numerical computations in finance (Tavella and Randall, 2000). After a linear system is generated from the discretization process, the iterative method is computed to solve the linear system. Then, several numerical experiments involving FSGS, HSGS, QSGS and QSMGS methods are executed to verify the effectiveness of QSMGS method.

\section{MATERIALS AND METHODS}

\section{Quarter-sweep Crank-Nicolson discretization} scheme: We describe the full-, half- and quarter-sweep Crank-Nicolson schemes in discretizing Eq. 1. Both the full and quarter-sweep Crank Nicolson approximation equations can be derived as Eq. 7:

$$
\begin{aligned}
& \frac{v_{i, j, k+1}-v_{i, j, k}}{\Delta t}= \\
& -\sigma_{1}^{2}\left(x_{0}+i p \Delta x\right)^{2}\left(\begin{array}{l}
\frac{v_{i-p, j, k}-2 v_{i, j, k}+v_{i+p, j, k}}{+v_{i-p, j, k+1}-2 v_{i, j, k+1}+v_{i+p, j, k+1}} \\
4(p \Delta x)^{2}
\end{array}\right) \\
& -\sigma_{2}^{2}\left(y_{0}+j p \Delta y\right)^{2}\left(\begin{array}{l}
\frac{v_{i, j-p, k}-2 v_{i, j, j}+v_{i, j+p, k}}{4}-2 v_{i, j, k+1}+v_{i, j+p, k+1} \\
4(p \Delta y)^{2}
\end{array}\right) \\
& -\rho \sigma_{1} \sigma_{2}\left(x_{0}+i p \Delta x\right)\left(y_{0}+j p \Delta y\right) \\
& \left(\begin{array}{l}
v_{i+p, j+p, k}+v_{i-p, j-p, k}-v_{i-p, j+p, k}-v_{i+p, j-p, k} \\
+v_{i+p, j+p, k+1}+v_{i-p, j-p, k+1}-v_{i-p, j+p, k+1}-v_{i+p, j-p, k+1} \\
8(p \Delta x)(p \Delta y)
\end{array}\right) \\
& \left.\begin{array}{l}
-r\left(x_{0}+i p \Delta x\right)\left(\frac{v_{i+p, j, k}-v_{i-p, j, k}+v_{i+p, j, k+1}-v_{i-p, j, k+1}}{4 p \Delta x}\right. \\
+r\left(y_{0}+j p \Delta y\right)\left(\frac{v_{i, j+p, k}-v_{i, j-p, k}+v_{i, j+p, k+1}-v_{i, j-p, k+1}}{4 p \Delta x}\right.
\end{array}\right) \\
& \left.\begin{array}{l}
v_{i, j, k}+v_{i, j, k+1} \\
2
\end{array}\right)
\end{aligned}
$$

Where:

$$
\begin{aligned}
& x=s_{1} \\
& y=s_{2}
\end{aligned}
$$

As $\mathrm{p}=1$ or $\mathrm{p}=2$, it represents full- and quartersweep schemes respectively.

Based on Eq. 7, the full-sweep Crank-Nicolson approximation equation is a nine-point finite difference scheme. Hence, to derive the half-sweep CrankNicolson, we rotate the entire axis clockwise by $45^{\circ}$ (Ali and Ling, 2008). As a result, a rotated nine-point approximation equation can be developed as Eq. 8: 


$$
\begin{aligned}
& \frac{v_{i, j, k+1}-v_{i, j, k}}{\Delta t}= \\
& -\sigma_{1}^{2}\left(x_{0}+i \Delta x\right)^{2}\left(\begin{array}{l}
v_{i-1, j+1, k}-2 v_{i, j, k}+v_{i+1, j-1, k} \\
+\frac{v_{i-1, j+1, k+1}-2 v_{i, j, k+1}+v_{i+1, j-1, k+1}}{4(\Delta x)^{2}}
\end{array}\right) \\
& -\sigma_{2}^{2}\left(y_{0}+j \Delta y\right)^{2}\left(\begin{array}{l}
v_{i+1, j+1, k}-2 v_{i, j, k}+v_{i-1, j-1, k} \\
\frac{+v_{i+1, j+1, k+1}-2 v_{i, j, k+1}+v_{i-1, j-1, k+1}}{4(\Delta y)^{2}}
\end{array}\right) \\
& -\rho \sigma_{1} \sigma_{2}\left(x_{0}+i \Delta x\right)\left(y_{0}+j \Delta y\right) \\
& \left(\begin{array}{l}
\mathrm{v}_{\mathrm{i}+2, \mathrm{j}, \mathrm{k}}+\mathrm{v}_{\mathrm{i}-2, \mathrm{j}, \mathrm{k}}-\mathrm{v}_{\mathrm{i}, j+2, \mathrm{k}}-\mathrm{v}_{\mathrm{i}, \mathrm{j}-2, \mathrm{k}} \\
+\frac{+\mathrm{v}_{\mathrm{i}+2, \mathrm{j}, \mathrm{k}+1}+\mathrm{v}_{\mathrm{i}-2, \mathrm{j}, \mathrm{k}+1}-\mathrm{v}_{\mathrm{i}, \mathrm{j}+2, \mathrm{k}+1}-\mathrm{v}_{\mathrm{i}, \mathrm{j}-2, \mathrm{k}+1}}{16(\Delta \mathrm{x})(\Delta \mathrm{y})}
\end{array}\right) \\
& -r\left(x_{0}+i \Delta x\right)\left(\begin{array}{l}
v_{i+1, j-1, k}-v_{i-1, j+1, k} \\
\frac{+v_{i+1, j-1, k+1}-v_{i-1, j+1, k+1}}{4 \sqrt{2} \Delta x}
\end{array}\right) \\
& -r\left(y_{0}+j \Delta y\right)\left(\begin{array}{l}
v_{i+1, j+1, k}-v_{i-1, j-1, k} \\
+v_{i+1, j+1, k+1}-v_{i-1, j-1, k+1} \\
4 \sqrt{2} \Delta x
\end{array}\right) \\
& +r\left(\frac{v_{i, j, k}+v_{i, j, k+1}}{2}\right)
\end{aligned}
$$

The nine-point approximation scheme for the full-, half- and quarter-sweep approaches can be illustrated in Fig. 1. According to Fig. 1, the implementations of FSGS, HSGS, QSGS and QSMGS iterative methods are performed onto the solid node points only until convergence criterion is met. Then, the remaining points are computed using direct method; see (Othman and Abdullah, 2000; Sulaiman et al., 2004a; 2004b; Koh and Sulaiman, 2009). Since half- and quartersweep approaches compute only half and quarter of the entire node points, theoretically they are faster than the standard full-sweep approach.

Modified Gauss-Seidel iterative methods: The approximations in Eq. 7 and 8 will generate large sparse linear system of form:

$$
A v=f
$$

Where:

$\mathrm{A}=$ Coefficient matrix

$\mathrm{f}=$ Known column vector, computed from the previous time level

$\mathrm{v}=$ Unknown column vector, values at the current time level

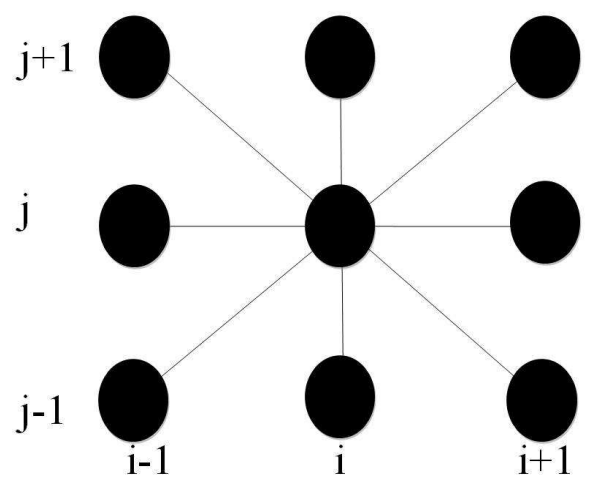

(a)

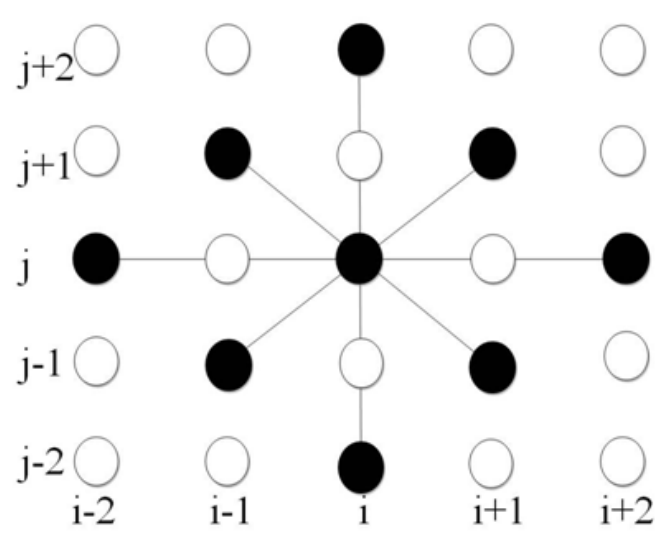

(b)

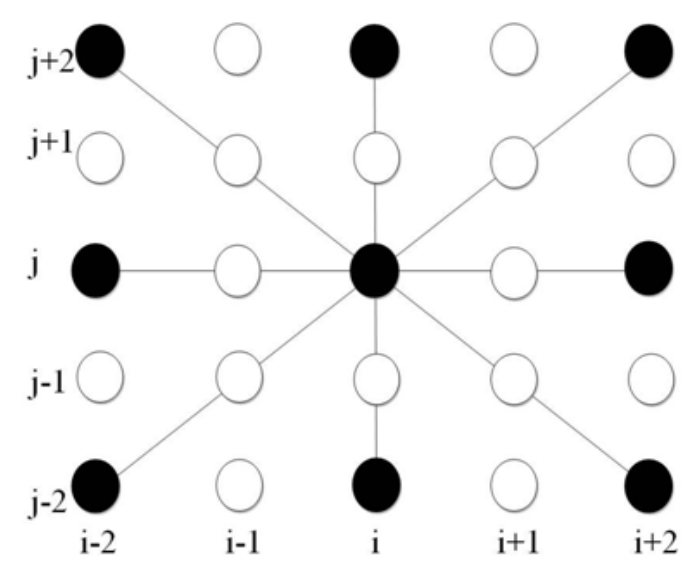

(c)

Fig. 1: Computational molecules of (a) full-sweep, (b) half-sweep and (c) quarter-sweep cases 
In general, the linear system in Eq. 9 can be solved by applying Algorithm 1 (GS method).

\section{Algorithm 1 (GS method):}

- Initializing all the parameters. Set $\mathrm{k}=0$

- General iteration:

$$
\mathrm{v}_{\mathrm{i}}^{(k+1)}=\frac{1}{\mathrm{~A}_{\mathrm{ii}}}\left(\mathrm{f}_{\mathrm{i}}-\sum_{\mathrm{j}=1}^{\mathrm{i}-1} \mathrm{~A}_{\mathrm{ij}} \mathrm{v}_{\mathrm{j}}^{(k+1)}-\sum_{\mathrm{j}=\mathrm{i}+1}^{\mathrm{i}-\mathrm{n}} \mathrm{A}_{\mathrm{ij}} \mathrm{v}_{\mathrm{j}}^{(k)}\right)
$$

- Convergence test:

If the error tolerance is fulfilled, the value option at that time level is $v_{i}^{(k+1)}$ and the algorithm stops. Else, set $\mathrm{k}=\mathrm{k}+1$ and go to step ii.

In addition, Gunawardena et al. (1991) proposed the Modified Gauss-Seidel (MGS) method which uses a preconditioned matrix. The matrix when multiplied with the coefficient matrix will be able to transform the upper codiagonal to zero (Gunawardena et al., 1991). Following to that, we apply the preconditioned matrix to Eq. 9 and get the subsequent preconditioned linear system Eq. 10:

$$
\mathrm{A} * \mathrm{v}=\mathrm{f} *
$$

Where:

$$
\begin{aligned}
& \mathrm{A}^{*}=\mathrm{PA} \\
& \mathrm{f}^{*}=\mathrm{Pf} \\
& \mathrm{P}=\mathrm{I}+\mathrm{S}
\end{aligned}
$$$$
S=\left(\begin{array}{ccccc}
0 & -\frac{a_{12}}{a_{11}} & 0 & \cdots & 0 \\
0 & 0 & -\frac{a_{23}}{a_{22}} & \cdots & 0 \\
\vdots & \vdots & \vdots & \ddots & \vdots \\
0 & 0 & 0 & \cdots & -\frac{a_{n-1 n}}{a_{n-\ln -1}} \\
0 & 0 & 0 & \cdots & 0
\end{array}\right)_{(n \times n)}
$$

I = Identity matrix

Then, the algorithm of MGS method can be formulated as in Algorithm 2.

\section{Algorithm 2 (MGS method):}

- Initializing all the parameters. Set $\mathrm{k}=0$.

- General iteration:

- $\quad \mathrm{v}_{\mathrm{i}}^{(k+1)}=\frac{1}{\mathrm{~A} *_{\mathrm{ii}}}\left(\mathrm{f} *_{\mathrm{i}}-\sum_{\mathrm{j}=1}^{\mathrm{i}-1} \mathrm{~A} *_{\mathrm{ij}} \mathrm{v}_{\mathrm{j}}^{(\mathrm{k}+1)}-\sum_{\mathrm{j}=\mathrm{i}+1}^{\mathrm{i}-\mathrm{n}} \mathrm{A} *_{\mathrm{ij}} \mathrm{v}_{\mathrm{j}}^{(k)}\right)$

- Convergence test:

If the error tolerance is fulfilled, the value option at that time level is $v_{i}^{(k+1)}$ and the algorithm stops.

Else, set $\mathrm{k}=\mathrm{k}+1$ and go to step ii.

\section{RESULTS}

Several numerical experiments are performed to test the effectiveness of FSGS, HSGS, QSGS and QSMGS iterative methods. And the exact solution for problem 1 is given by Eq. 11 Haug (2007):

$$
\begin{aligned}
\mathrm{v}\left(\mathrm{s}_{1}, \mathrm{~s}_{2}, \mathrm{~T}\right) & =\mathrm{s}_{1} \mathrm{M}\left(\varphi_{1}, \mathrm{~d} ; \rho_{1}\right)+\mathrm{s}_{2} \mathrm{M}\left(\varphi_{2},-\mathrm{d}+\sigma \sqrt{\mathrm{T}} ; \rho_{2}\right) \\
& -\mathrm{Ke}^{-\mathrm{rT}}\left[1-\mathrm{M}\left(-\varphi_{1}+\sigma_{1} \sqrt{\mathrm{T}},-\varphi_{2}+\sigma_{2} \sqrt{\mathrm{T}} ; \rho\right)\right]
\end{aligned}
$$

Where:

$$
\mathrm{d}_{1}=\frac{\ln \left(\frac{\mathrm{s}_{1}}{\mathrm{~s}_{2}}\right)+\left(\frac{\sigma^{2}}{2}\right) \mathrm{T}}{\sigma \sqrt{\mathrm{T}}}
$$

$\varphi_{1}=\frac{\ln \left(\frac{\mathrm{s}_{1}}{\mathrm{~K}}\right)+\left(\frac{\mathrm{r}+\sigma_{1}^{2}}{2}\right) \mathrm{T}}{\sigma_{1} \sqrt{\mathrm{T}}}$

$\varphi_{2}=\frac{\ln \left(\frac{\mathrm{s}_{2}}{\mathrm{~K}}\right)-\left(\frac{\mathrm{r}+\sigma_{2}^{2}}{2}\right) \mathrm{T}}{\sigma_{2} \sqrt{\mathrm{T}}}$

$\sigma=\sqrt{\sigma_{1}^{2}+\sigma_{2}^{2}-2 \rho \sigma_{1} \sigma_{2}}$

$\rho_{1}=\frac{\sigma_{1}-\rho \sigma_{2}}{\sigma}$ 
J. Math. \& Stat., 8 (1): 129-135, 2012

Table 1: Number of iterations, execution time and RMSE for FSGS, HSGS, QSGS and QSMGS methods

\begin{tabular}{|c|c|c|c|c|c|c|c|c|c|c|c|c|}
\hline \multirow[b]{3}{*}{ Mesh size } & \multicolumn{12}{|c|}{ Method } \\
\hline & \multicolumn{3}{|c|}{ FSGS } & \multicolumn{3}{|c|}{ HSGS } & \multicolumn{3}{|c|}{ QSGS } & \multicolumn{3}{|c|}{ QMSGS } \\
\hline & Iter. & Time(s) & RMSE & Iter. & Time(s) & RMSE & Iter. & Time(s) & RMSE & Iter. & Time(s) & RMSE \\
\hline$\overline{50}$ & 18 & 0.43 & $1.05 \mathrm{e}-02$ & 14 & 0.25 & $1.29 \mathrm{e}-01$ & 11 & 0.15 & $8.63 \mathrm{e}-02$ & 10 & 0.14 & $8.63 \mathrm{e}-02$ \\
\hline 100 & 41 & 3.23 & $2.47 \mathrm{e}-03$ & 27 & 1.48 & $1.26 \mathrm{e}-01$ & 18 & 0.53 & $2.37 \mathrm{e}-02$ & 15 & 0.47 & $2.37 \mathrm{e}-02$ \\
\hline 150 & 76 & 13.43 & $8.74 \mathrm{e}-04$ & 46 & 5.35 & $1.21 \mathrm{e}-01$ & 28 & 1.48 & $7.07 \mathrm{e}-03$ & 21 & 1.23 & $7.07 \mathrm{e}-03$ \\
\hline 200 & 124 & 48.91 & $6.48 \mathrm{e}-04$ & 70 & 16.75 & $1.17 \mathrm{e}-01$ & 41 & 3.67 & $5.74 \mathrm{e}-03$ & 30 & 2.85 & $5.74 \mathrm{e}-03$ \\
\hline 250 & 185 & 108.45 & $4.05 \mathrm{e}-04$ & 102 & 38.98 & $1.14 \mathrm{e}-01$ & 57 & 8.14 & $3.77 \mathrm{e}-03$ & 41 & 6.08 & $3.77 \mathrm{e}-03$ \\
\hline 300 & 259 & 220.49 & $2.21 \mathrm{e}-04$ & 140 & 78.00 & $1.12 \mathrm{e}-01$ & 76 & 15.91 & $1.78 \mathrm{e}-03$ & 54 & 11.58 & $1.78 \mathrm{e}-03$ \\
\hline 350 & 345 & 419.78 & $2.11 \mathrm{e}-04$ & 184 & 139.42 & $1.11 \mathrm{e}-01$ & 98 & 27.43 & $1.89 \mathrm{e}-03$ & 69 & 19.70 & $1.89 \mathrm{e}-03$ \\
\hline
\end{tabular}

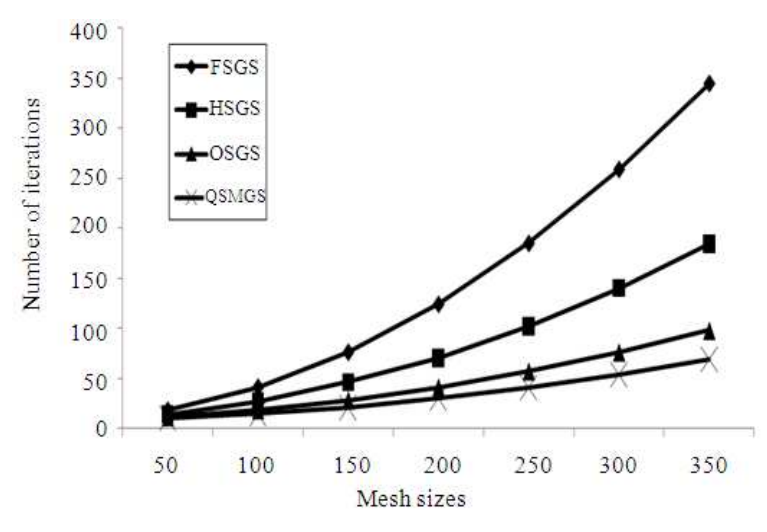

Fig. 2: Number of iterations versus mesh sizes of FSGS, HSGS, QSGS and QSMGS methods

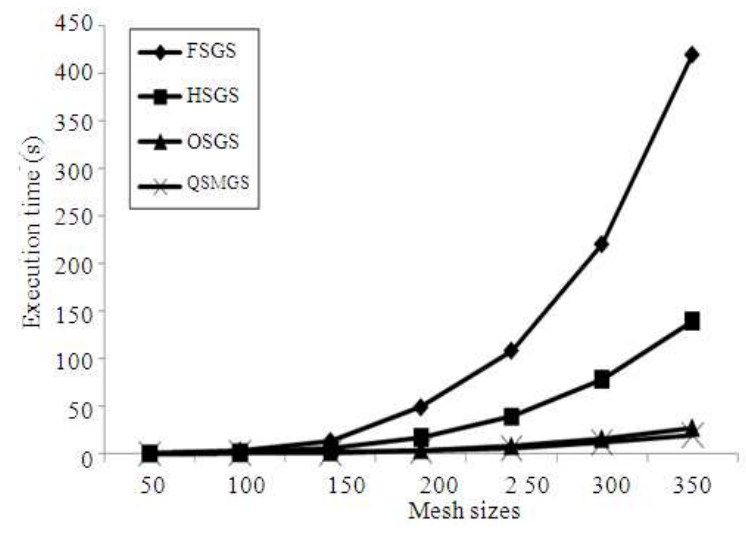

Fig. 3: Execution time (s) versus mesh sizes of FSGS, HSGS, QSGS and QSMGS methods

$\mathrm{M}(\mathrm{a}, \mathrm{b} ; \rho)$ Represents the cumulative bivariate normal distribution function; see (Drezner and Wesolowsky, 1990; Haug, 2007).

The parameters considered are $\mathrm{T}=0.1, \mathrm{~K}=100, \mathrm{r}=$ $0.03, \sigma_{1}=0.5, \sigma_{2}=0.5, \rho=0.5, s_{1}=[0,300]$ and $s_{2}=$ [0,300] which are also used by Jeong et al. (2009).
Nonetheless in this study, we have 100 time steps with the tested matrix sizes, 50, 100, 150, 200, 250, 300 and 350. Furthermore, ET $=10^{-10}$ will be the error tolerance. In computational finance, Root Mean Squared Relative Error (RMSE) is widely used to assess the accuracy of the iterative solutions such as in Zhao et al. (2007), Jeong et al. (2009) and Koh et al. (2010a). The RMSE is defined by:

$$
\operatorname{RMSE}=\sqrt{\frac{1}{\left(\mathrm{~N}_{\mathrm{x}}-1\right)\left(\mathrm{N}_{\mathrm{y}}-1\right)} \sum_{\mathrm{i}=1}^{N_{\mathrm{x}}} \sum_{\mathrm{j}=1}^{N_{\mathrm{y}}}\left(\frac{\tilde{\mathrm{v}}_{\mathrm{i}, \mathrm{j}}-\mathrm{v}_{\mathrm{i}, \mathrm{j}}}{\varepsilon+\mathrm{v}_{\mathrm{i}, \mathrm{j}}}\right)^{2}}
$$

Where:

$\mathrm{v}_{\mathrm{i}, \mathrm{j}}=$ Exact value

$\mathrm{v}_{\mathrm{i}, \mathrm{j}}=$ Numerical value

$\varepsilon=$ Small positive number to avoid dividing by a too small number

$\mathrm{N}_{\mathrm{x}}=$ Mesh size for $\mathrm{x}$

$\mathrm{N}_{\mathrm{y}}=$ Mesh size for $\mathrm{y}$

These computational experiments are implemented in the Intel Core 2 Duo, $2.93 \mathrm{GHz}$ processor. The computational results for FSGS, HSGS, QSGS and QSMS are tabulated in Table 1 and graphically displayed in Fig. 2 and 3.

\section{DISCUSSION}

The numerical experiments analyze FSGS, HSGS, QSGS and QSMGS from the aspects of number of iterations, execution time and RMSE. Based on the numerical results presented in Table 1 as well as Fig. 2 and 3 they clearly show that QSMGS method has the least number of iterations and execution time among the tested iterative methods. The number of iterations for HSGS, QSGS and QSMGS methods reduces by 22.22$44.67 \%, 38.89-71.59 \%$ and $44.44-80.00 \%$ respectively compared to FSGS method. In terms of execution time, HSGS, QSGS and QSMGS methods speed up 
approximately $41.86-66.79 \%, 65.12-93.47 \%$ and $67.44-$ $95.31 \%$ respectively relative to FSGS method. On the other hand, the accuracies of the iterative methods are in good agreement except for HSGS which is not so fine.

\section{CONCLUSION}

In this study, we examined the application of QSMGS iterative method in evaluating European option with two-underlying assets by solving the twodimensional Black-Scholes PDE. From the results presented, they can be observed that QSMGS iterative method converges faster than the other methods by having least number of iterations. Besides that, QSMGS manages to retain the accuracy of the standard GaussSeidel iterative method. Thus, we can conclude that QSMGS method is a better method compared to HSGS or FSGS method in the sense of number of iterations and execution time.

For future works, other types of option pricing, for example American option with two underlying assets or even other exotic options can be explored through the applications of quarter-sweep approach. In the context of iterative method, we can improve the preconditioned iterative method by employing the Improving Modified Gauss-Seidel method, (Kohno et al., 1997) which had been applied in solving one-dimensional European option pricing (Koh et al., 2010b). Also, family of twostage iterative methods, for example AGE (Evans and Sahimi, 1988), IADE (Sahimi et al., 1993), HSIADE (Sulaiman et al., 2004a), QSIADE (Sulaiman et al., 2004b), AM (Ruggiero and Galligani, 1990), HSAM (Sulaiman et al., 2004c) and QSAM (Muthuvalu and Sulaiman, 2010) can be considered with the preconditioned iteration concept.

\section{ACKNOWLEDGMENT}

This study was supported by Postgraduate Research Grant (GPS0004-SG-1/2009), Universiti Malaysia Sabah, Kota Kinabalu, Sabah, Malaysia.

\section{REFERENCES}

Ali, N.H.M. and S.T. Ling, 2008. Rotated Krylov preconditioned iterative schemes in the solution of convection-diffusion equations. Applied Math. Comput., 206: 425-437. DOI: 10.1016/j.amc.2008.09.023

Black, F. and M. Scholes, 1973. The pricing of options and corporate liabilities. J. Political Econ., 81: 637654. DOI: $10.1086 / 260062$
Drezner, Z. and G.O. Wesolowsky, 1990. On the computational of the bivariate normal integral. J. Stat. Comput. Simul., 35: 101-107. DOI: 10.1080/00949659008811236

Evans, D.J. and M.S. Sahimi, 1988. The Alternating Group Explicit (AGE) iterative method for solving parabolic equations 1: 2-dimensional problems. Int. J. Comput. Math., 24: 311-341. DOI: 10.1080/00207168808803651

Gunawardena, A.D., S.K. Jain and L. Snyder, 1991. Modified iterative methods for consistent linear systems. Linear Algebra Appli., 154-156: 123-143. DOI: 10.1016/0024-3795(91)90376-8

Haug, E.G. 2007. The Complete Guide to Option Pricing Formulas. 2nd Edn., McGraw-Hill, New York, ISBN-10: 0071389970, pp: 536.

Jeong, D., J. Kim and I.S. Wee, 2009. An accurate and efficient numerical method for Black-Scholes equations. Commun. Korean Math. Soc., 24: 617628. DOI: 10.4134/CKMS.2009.24.4.617

Koh, W.S. and J. Sulaiman, 2009. Quarter sweep modified gauss-seidel method using Crank-Nicolson approach for European put option pricing. Proceedings of the 5th Asian Mathematical Conference, (AMC' 09), Kuala Lumpur, pp: 261-267.

Koh, W.S., J. Sulaiman and R. Mail, 2010a. Quartersweep projected modified Gauss-Seidel algorithm applied to linear complementarity problem. Am. J. Applied Sci., 7: 790-794. DOI: 10.3844/ajassp.2010.790.794

Koh, W.S., J. Sulaiman and R. Mail, 2010b. Quartersweep improving modified Gauss-Seidel method for pricing European Option. MATEMATIKA, 26: 179-185.

Kohno, T., H. Kotakemori, H. Niki and M. Usui, 1997. Improving the modified gauss-seidel method for $Z$ matrices. Linear Algebra Appli., 267: 113-123. DOI: $10.1016 /$ S0024-3795(97)80045-6

Merton, R.C., 1973. Theory of rational option pricing. Bell J. Econ. Manage. Sci., 4: 141-183. DOI: $10.2307 / 3003143$

Muthuvalu, M.S. and J. Sulaiman, 2010. Quarter-Sweep Arithmetic Mean (QSAM) iterative method for second kind linear fredholm integral equations. Applied Math. Sci., 4: 2943-2953.

Othman, M. and A.R. Abdullah, 2000. An efficient Four Points Modified Explicit Group Poisson solver. Int. J. Comput. Math., 76: 203-217. DOI: 10.1080/00207160008805020

Ruggiero, V. And E. Galligani, 1990. An iterative method for large sparse systems on a vector computer. Comput. Math. Appli., 20: 25-28. DOI: 10.1016/0898-1221(90)90065-R 
Sahimi, M.S., A. Ahmad, A.A. Bakar, 1993. The Iterative Alternating Decomposition Explicit (IADE) method to solve the heat conduction equation. Int. J. Comput. Math., 47: 219-229. DOI: $10.1080 / 00207169308804179$

Stulz, R.M., 1982. Options on the minimum or the maximum of two risky assets: Analysis and applications. J. Finan. Econ., 10: 161-185. DOI: 10.1016/0304-405X(82)90011-3

Sulaiman, J., M. Othman and M.K. Hasan, 2004a. The Half-Sweep Iterative Alternating Decomposition Explicit (HSIADE) method for diffusion equation. Comput. Inform. Sci., 3314: 57-63. DOI: 10.1007/978-3-540-30497-5_10

Sulaiman, J., M. Othman and M.K. Hasan, 2004b. Quarter-sweep iterative alternating decomposition explicit algorithm applied to diffusion equations. Int. J. Comput. Math., 81: 1559-1565. DOI: $10.1080 / 00207160412331291125$
Sulaiman, J., M. Othman and M.K. Hasan, 2004c. A new Half-Sweep Arithmetic Mean (HSAM) algorithm for two-point boundary value problems. Proceedings of the International Conference on tatistics and Mathematics and its Application in the Development of Science and Technology, (ADST' 04), Bandung, Indonesia, pp: 169-173.

Tavella, D. and C. Randall, 2000. Pricing Financial Instruments: The Finite Difference Method. 1st Edn., John Wiley and Sons, New York, ISBN: 0471197602, pp. 237.

Zhao J., M. Davison and R.M. Corless, 2007. Compact finite difference method for American option pricing. J. Comput. Applied Math., 206: 306-321. DOI:10.1016/j.cam.2006.07.006 